\title{
L'ailleurs du réel
}

Chantal Lapeyre-Desmaison

Université d'Artois

L'état du récit aujourd'hui, ses métamorphoses comme son devenir, ou ses impasses, doivent être envisagés dans tous les lieux où ils se manifestent : dans la littérature contemporaine certes, ou dans ce qu'on nomme hâtivement peut-être la paralittérature, mais aussi dans le cinéma et dans les séries télévisées, dont l'expansion me paraît significative de l'engouement qu'elles suscitent. La série américaine $X$-Files est à ce titre un objet d'étude qui mérite considération. Cette série en 202 épisodes, créée par Chris Carter et diffusée à partir de 1993 aux États-Unis et de 1994 en France, a rencontré très vite un grand succès et elle a obtenu plusieurs Emmy Awards et Golden Globe Awards. À mes yeux, elle appartient à un 
ensemble de créations dans le champ télévisuel et cinématographique qui témoignent d'une manière fine et subtile de ce qu'est aujourd'hui l'imaginaire contemporain. C'est pourquoi il m'a paru pertinent, dans le cadre d'un dossier consacré $\mathrm{au}$ "réel $\mathrm{du}$ récit/ récit du réel», de réfléchir à la manière dont la fiction contemporaine la plus visible, et la plus immédiatement lisible, comme la donne à lire une série grand public, informe des formes et des enjeux de cet imaginaire. En même temps, bien que cette série n'ait pas d'incidences manifestes sur la production littéraire - outre l'inévitable phénomène de novellisation et l'écriture de quelques romans reprenant l'univers de la série, mais avec des intrigues originales - il ne me semble pas impossible de la rapprocher d'un ensemble d'œuvres littéraires contemporaines qui reposent sur des principes identiques quant à l'approche du réel et de la réalité. Il me semble en effet que cette série pose de manière critique la question des rapports qu'entretiennent la fiction et la réalité, le récit et le réel dans la création contemporaine. Indirectement, $X$-Files, série-symptôme de l'époque qui l'a vue naître, permet ainsi de penser ce qui est aussi en jeu dans une certaine production littéraire contemporaine, pour qui le réel est à la fois cause et visée de l'écriture.

\section{Un symptôme de l'imaginaire contemporain}

Le corpus retenu pour la présente étude est constitué des dix premiers épisodes de la saison 1, incluant donc le pilote, épisode qui, traditionnellement, sert à lancer une série et permet aux producteurs de savoir si le projet a un avenir. Or, ces premiers épisodes ont reçu une audience importante. Si $X$ - 
Files a frappé les esprits au moment de la diffusion, c'est sans doute lié à un ensemble de facteurs qui incluent à la fois les thèmes retenus et les modalités narratives : la série repose sur des principes de narration aux vertus éprouvées, elle met en scène des personnages au dessin complexe et l'histoire racontée ne se laisse pas aussi aisément réduire à quelques schémas simplistes jouant des images conventionnelles dans le domaine du fantastique que les médias ont pu le laisser supposer. Les deux premiers épisodes instituent les fondations thématiques de la série à partir de cette évidence qui isole l'agent Mulder de ses collègues et du monde auquel il appartient pourtant: Nous ne sommes pas seuls, dit le titre du premier épisode. Pour Mulder en effet, le monde ne se résume pas à ce que nous en percevons, à ce qu'on nous en dit $^{1}$. À cet égard la série témoigne, au moins en apparence, de cet « imaginaire du complot mondial » dont Pierre-André Taguieff a bien montré la structure et les enjeux politiques et éthiques. En outre, le réemploi constant de certaines figures essentielles dans le domaine du fantastique (fantômes, goules, prédateurs archaïques par exemple) assure à la série, en tension avec la question des extraterrestres, sa cohérence profonde. Parmi les dix premiers épisodes de la saison 1, on note en effet au moins trois épisodes qui ne sont pas liés aux extraterrestres et à leurs accointances gouvernementales. Dans Compressions, Mulder et

1 Gorge profonde, le second épisode au titre repris du surnom donné à l'informateur secret des deux journalistes lors du scandale du Watergate, met en scène un homme qui aidera tout aussi secrètement Mulder dans ses enquêtes. On retrouve cette même problématique dans L'enlèvement, Espace, Ange déchu (épisodes 4, 9 et 10) : un OVNI qui s'abat dans une forêt du Wisconsin, des enlèvements par les extraterrestres, un colonel de la Nasa habité par une entité hostile qui le pousse au sabotage des navettes envoyées dans l'espace. 
Scully enquêtent sur des meurtres inexpliqués : on trouve les corps dans des pièces hermétiquement closes, personne n'a pu entrer ou sortir, et le foie de la victime a été dévoré. Le meurtrier est en réalité un être énigmatique qui hiberne depuis des années (des siècles peut-être) et qui, tous les trente ans, sort de son nid pour se nourrir de foies humains aux vertus régénératrices. S'il parvient à tuer sans laisser de traces, c'est parce que son corps est doté d'une extrême élasticité qui lui permet de se glisser dans les conduits d'aération les plus étroits. C'est un prédateur au regard inquiétant qui rassemble en lui tous les traits des figures effrayantes dont la tradition littéraire est riche, comme les goules ou les vampires. Le diable du New Jersey conduit Mulder et Scully à découvrir la persistance du plus archaïque dans le présent le plus actuel : des êtres quelque peu simiesques ou néanderthaliens vivent dans les bois du Jersey et survivent en chassant toutes sortes de proies, y compris des proies humaines. Ce qui ne semblait qu'être légende s'avère : notre civilisation n'est qu'apparence, le passé le plus ancien, illustré ici par les pratiques anthropophages des sauvages, est toujours vif, toujours présent, et il est susceptible de surgir et d'attaquer. Peut-être est-ce ce qui soudainement fascine Mulder quand il rencontre la femelle et se déclare étourdi par sa beauté. Enfin, la mort même est incertaine. Certitude tangible de nos civilisations, elle est mise en doute dans l'épisode intitulé L'ombre de la mort. Comme dans de nombreux contes ou nouvelles, un homme assassiné cherche à obtenir justice en se manifestant par la télékinésie auprès d'une jeune femme qu'il aimait comme sa fille adoptive. C'est un des premiers sens qu'on peut donner au sous-titre français de la série Aux frontières du réel : la violence archaïque, la révolte de l'innocence bafouée, les nécessités 
vitales les plus élémentaires, si elles ont été rejetées aux frontières du réel, aux frontières de notre réalité la plus banale, la plus quotidienne, n'ont jamais disparu : elles persistent sous les comportements les plus civilisés. En apparence. C'est aussi ce que sait Mulder et qui l'isole de ses contemporains. Deux autres épisodes ont un statut plus complexe dans la mesure où ils lient ensemble les deux pôles : politique fiction si l'on veut et syncrétisme des références légendaires et/ou mythiques. Dans Un fantôme dans l'ordinateur, un savant a créé un ordinateur extraordinairement perfectionné dont la maîtrise lui échappe (on retrouve ici le fil de Mary Shelley), mais le gouvernement empêche toute tentative de remédiation à cette monstruosité. Les agents Mulder et Scully auront donc à combattre à la fois la folie de la machine, née de l'orgueil et de la folie humaine, mais aussi les agents gouvernementaux qui ont intérêt à sa survie. Dans Projet arctique, des scientifiques qui foraient la calotte glaciaire sont retrouvés morts: ils se sont ou suicidés ou entretués. En réalité, Mulder et Scully, dépêchés sur place avec une équipe de scientifiques, s'aperçoivent qu'ils ont été contaminés par un ver vivant depuis 25000 ans dans la glace et qui, se fixant dans l'hypothalamus, accroît l'agressivité et la violence de son hôte. Le gouvernement intervient à la fin de l'épisode pour raser la base scientifique, interdisant ainsi à Mulder de réaliser son projet : inspecter attentivement le site et enquêter sur ce ver mystérieux qui pourrait bien être d'origine extraterrestre. L'épisode mêle donc les deux axes précédemment évoqués : le passé dévoile sa vérité violente et insiste, avec une force dévastatrice dans le présent, mais ce savoir est dénié par ceux-là même qu'intéressent, pour des motifs économiques et politiques, sa dissolution, sa révocation. 
Consentir en effet à cette évidence qui s'avère par le biais de la science ferait encourir des risques vitaux à la civilisation.

Ces fils thématiques qui se nouent dès les premiers épisodes placent le spectateur dans une curieuse position, entre reconnaissance - à plusieurs niveaux - et fascination (parce que ce qui est reconnu ne l'est toujours que partiellement et que la temporalité de la réception, sa fluidité, ne permettent pas l'arrêt méditatif, le temps du retour qu'autorise la lecture quand elle se fait décision). Le spectateur est donc incessamment pris dans un mouvement de reconnaissance et d'illusions. Il est comme le lecteur que définit ainsi Iser : «Si les objets que l'on imagine au cours de la lecture se caractérisent par le fait de donner une présence à ce qui n'est pas donné ou à ce qui est absent, cela veut dire que nous nous trouvons face à une représentation, c'est vivre une irréalisation. » (1997, p. 253) Cette irréalisation, propre à l'expérience de lecture des systèmes sémiotiques, est littéralement captivante. Mais elle l'est d'autant plus que les principes de narration mis en œuvre privilégient toujours le mi-dire: la série est complexe parce qu'elle élude les liens logiques d'une thématique à une autre, mais aussi d'un épisode à l'autre, et d'une scène à l'autre. C'est au spectateur qu'incombe la tâche de restituer les liens manquants, de combler la vacuité du blanc dans le tissu fictionnel. Ce phénomène est particulièrement sensible dans l'épisode consacré au très curieux Eugen Tooms, qui hiberne pendant trente années avant de ressurgir pour dévorer des foies humains. Certes, des hypothèses sont émises quant à son origine (un mutant, suggère Scully à un moment), mais jamais aucune vérité sur les origines du personnage, sur ses motivations n'est dévoilée. Il existe dans une matité absolue. De même, l'épisode s'achève sans aucune résolution : un gardien 
apporte son repas à Tooms, qui lèche des morceaux de papier, et la dernière scène montre le personnage regardant la fente du passe-plat. On devine que cette fin n'en est pas une, que les Tooms, sous cette forme ou une autre, ressurgiront, mais on ne sait ni comment ni, surtout, pourquoi.

Si la série s'avère symptomatique, elle le doit donc à cette manière de traiter, dans un syncrétisme constant, des apports mythologiques d'origines variées, et elle le doit aussi à cette pratique de la scansion énigmatique. Elle révèle à cet égard qu'une question essentielle hante la période contemporaine, touchant à la nature de la réalité, du réel et du langage dans cette entreprise. Le surgissement de cette série dans le paysage fictionnel contemporain n'est pas sans évoquer le XVIIIe siècle : en réponse (ou en réaction) aux dérives qu'a pu engendrer la pensée du siècle dit «des Lumières » est apparue la réaction illuministe. D'une lumière à l'autre... Tout se passe en effet comme si la volonté de faire la lumière, d'éclairer le monde à tout prix, engendrait une frustration accrue qui révèle par contrecoup la nécessité de l'ombre. La série $X$-Files apparaît dans ce contexte comme la résurrection opportune de l'ombre, dont seul Mulder pressentait l'existence, pour contrecarrer le dévoilement excessif qui s'avère n'être qu'une autre forme de voile, une forme plus pernicieuse encore.

Dans Bienvenue dans le désert du réel, Slavoj Zizek note, à la suite de Badiou :

L'expérience dernière du $\mathrm{XX}^{\mathrm{e}}$ siècle, permettant de le définir comme époque historique, aura été celle du réel, en tant que le réel ne se réduit pas à la réalité sociale, quotidienne, mais se conçoit dans son extrême violence comme ce qui reste après qu'on a dépouillé la réalité de son écorce trompeuse. (p. 24) 
De cette passion du réel, entendu en un sens large, la série $X$ Files me paraît également symptomatique dans la mesure où sa thématique principale prend appui sur des faits de société avérés : la théorie du complot occulte, très présente dans la série, rejoint, comme le montre Taguieff, des courants de pensée américains dont certains débouchent sur des actions terroristes contre un État perçu comme une entité maléfique, trompeuse et malveillante. Le réel, dans ce contexte, se définissant comme vérité dissimulée, occultée, c'est au nom de ce qui est tu, au nom de ce qui est caché que ces groupuscules d'extrême-droite interviennent de manière parfois sanglante ${ }^{2}$. C'est là tout le danger de cette passion du réel que dénonce Zizek dans son essai.

\section{Du trop de réalité dans $\mathrm{X}$-Files}

À condition de définir plus précisément le concept de réel, on peut dire en effet que l'objet véritable de la fiction dans cette série n'est autre que le réel, c'est-à-dire ce qui surgit quand on retraverse le fantasme (comme le suggère Lacan, la réalité, c'est le réel passé au tamis du fantasme). Ce serait là le réel auquel s'affronte Mulder, un réel abusivement tenu pour la réalité même, cette dernière se caractérisant en même temps par un excès et une pauvreté. Surabondante réalité : elle est tissée de tout ce que nos yeux perçoivent, de tous les objets qui nous entourent, de toutes les paroles qui nous bercent. Réalité qui a bien souvent les apparences d'un rêve éveillé et nous donne à voir et à penser un monde lisse, plein, sans solution de

2 Comme Timothy MacVeigh, auteur d'un attentat qui a causé 168 morts à Oklahoma City en 2001. 
continuité. Mais d'une extrême pauvreté en même temps: tissée du néant médiatique, d'images communes à tous, de piètres consolations. Mulder, dans ce contexte, à qui le sommeil de l'illusion est interdit, Mulder qui est assailli par les failles que peu d'humains sont capables de percevoir, doté d'une seconde vue que lui a octroyée un traumatisme vécu dans l'enfance, puisque sa sœur a disparu une nuit dans un éclat de lumière dont on ne sait s'il s'agit d'un souvenir écran ou d'une expérience réelle, Mulder donc serait un intégriste de ce réel dissimulé par un Autre foncièrement méchant.

Le problème essentiel concerne en effet la vérité et la possibilité d'y accéder, comme le suggère la phrase fétiche de la série : « La vérité est ailleurs». Pour Mulder, le langage et ses effets, corrélés à la question de la vérité, problématique obsédante, sont un vecteur de mensonges. Sa marginalité tient au fait qu'il ne se résout pas à croire que la vérité est tout entière là, voire qu'elle est toute. Ce qu'on voit et ce qu'on dit n'est pas ce qui est. Le sens de ses enquêtes gît en ce point : il s'agit toujours, au-delà de la quête de révélations, de souligner l'incomplétude foncière de ce qui est dit, de ce qui est montré. Et peut-être de ce qui est éprouvé, en tant que l'éprouvé est en lien étroit avec le langage qui le porte et le suscite. Pour lui, l'au-delà de la réalité abrite la vérité. Et cette vérité peut être nommée : ce sont ces "extraterrestres», les bien-nommés en effet, qui s'allient obscurément au monde du pouvoir. Que Mulder en soit réduit à donner des noms à ce que voile le symbolique, d'une manière plus ou moins convaincante, témoigne de la tension productive entre imaginaire et réel: l'imaginaire voulant nommer le réel imprenable au signifiant en propose alors une figuration, un récit, fiction véridique certes, mais qui doit être reconnue comme telle, ce que le personnage ne peut concevoir. 
Dans ce contexte, la critique de Zizek envers la passion du réel mérite considération :

Car le problème de la passion pour le réel, au XX $\mathrm{X}^{\mathrm{e}}$ siècle, n'est pas la passion pour le réel en tant que telle mais plutôt qu'il s'est agi d'une fausse passion, dont l'impitoyable quête, cette poursuite du réel derrière les apparences, était le stratagème suprême visant précisément à éviter sa rencontre. (p. 49)

La passion du réel qui affecte Mulder verse en effet, de par la nature même de ce réel, à une passion des semblants. Au fond, la thèse qui consiste à croire en l'existence d'un complot entre le pouvoir et les envahisseurs n'est qu'un nom, une image de plus, un semblant, visant à recouvrir le trou qui surgit dans le tissu même du savoir. Et ce semblant relève du symbolique pur, médiatisé par l'imaginaire. Nommer ce qui fait défaut dans le langage "complot» est un tour de passe-passe qui rabat l'imaginaire sur le symbolique, dans l'esquive la plus totale du réel qui, lui, appelle une pensée véritablement éthique. Il y aurait donc d'autres manières d'aborder ce réel, sinon de le traiter. Or, il me semble que la série a fait dans ce contexte l'objet d'un malentendu. On a trop souvent mis l'accent - et Taguieff n'y échappe pas - sur ce versant du complot sans tenir compte des épisodes, qui occupent pourtant une place importante, revitalisant les archétypes imaginaires du prédateur, du vampire, du sauvage ou du fantôme.

Le trait de génie des scénaristes, à cet égard, a été précisément de trouer une seconde fois le savoir troué que dissémine le langage par des représentations de l'irreprésentable. C'est en effet ainsi que je propose de comprendre le sens de ces épisodes qui semblent se dissocier de la trame principale consacrée au complot gouvernemental. Dans Compressions, Le diable du New Jersey ou L'ombre de la 
mort, quelque chose cherche à se dire, à se formuler et n'y parvient qu'en jouant des images archétypales dont l'imaginaire populaire est riche depuis toujours. Car le réel, ce qui n'a ni ordre ni sens, Lacan le rappelle, violence pure qui échappe à toute nomination, est une hantise comme le montre par exemple Eugen Tooms, cet être énigmatique qui ne cesse de revenir et qui se construit un nid d'abjection avec la bile qu'il consomme et de vieux papiers collés. Le fantôme de L'ombre de la mort qui revient, et reviendra longtemps, tant que les paroles qui libèrent n'ont pas été prononcées, rappelle que le réel - de la mort, du corps - , ce réel que la réalité voile tant bien que mal (et d'ailleurs trop bien pour Mulder) est toujours " gueule ouverte» :

Aucune interruption n'est possible, et l'espoir demeure vain d'accomplir enfin un acte d'une telle portée que le réel resterait enfin à sa place. Non, il restera toujours menaçant, féroce et jeune comme l'enfant dont il est le jumeau secret. Il ne laissera jamais s'installer un temps où chaque jour serait dimanche, où ne s'imposerait nul besoin de s'activer, de jouer, de travailler pour refouler! (Pommier, 2004, p. 53)

Cet inachèvement foncier dans la tâche, assignée à chacun, de circonvenir le réel pour simplement subsister donne la clé pour comprendre le principe de narration en œuvre dans X-Files, dont chaque épisode est caractérisé par son inachèvement : il n’y a pas de dernier mot possible contre le réel. Ce qui est hors sens le demeure. Jamais nous ne saurons pourquoi existe un Eugen Tooms; jamais on ne nous expliquera comment il est possible que des êtres sauvages, au comportement de Néanderthaliens, aient survécu dans les forêts du New Jersey, pas plus que nous ne saurons si réellement la télékinésie est l'arme de ceux qui n'ont pas trouvé le repos dans la mort. Chaque épisode montre un bout de réel - et Lacan, dans Joyce 
le sinthome, souligne le fait que "nous ne pouvons atteindre que des bouts de réel » (p. 123) - et ne peut que le montrer. Il est impuissant à en faire autre chose. Le réel qui surgit et vient trouer le tissu symbolique déployé par la fantasmagorie du complot rappelle le semblant à son ordre.

"I want to believe », dit une affiche dans le bureau de Mulder. Cette phrase pourrait être interprétée selon deux voies contraires : je veux croire ; je veux, comme les autres, être tissé de l'étoffe de mes songes; je veux rêver le même rêve que tous. Mais aussi : «je veux croire » qu'il existe autre chose ; je veux croire en un impossible, peut-être, que je pressens sans pouvoir le nommer, et je veux croire que cet impossible a un sens. Mulder, que cette passion du réel voue à la mélancolie, ne peut s'en tenir à la pauvreté, non du réel, mais de la réalité, et il pressent, de manière très lacanienne ou comme un amateur de la théorie de Gödel, qu'il faut supposer un au-delà de cette réalité qui lui donne sa consistance, mais qui reste indémontrable en son sein. Mais dans le même temps, il ne peut accepter qu'il n'y ait pas de dernier mot; il ne peut consentir à la défaillance foncière du sens et, en un geste aujourd'hui sans doute très polémique, il réaffirme la nécessité ontologique de la croyance.

À cet égard, les amateurs de la série qui insistent toujours sur le contraste entre les deux personnages principaux, le rêveur halluciné qu'est Mulder selon la logique qui le différencie des autres, et Scully, scientifique froidement rationnelle, n'accordent pas assez d'importance à ce détail : la jeune femme porte au cou une petite croix. Des épisodes ultérieurs évoqueront sa croyance en Dieu jamais reniée, sa foi en la religion catholique. Pour autant, s'agit-il d'une même 
croyance? Mulder est-il une figure du damné par manque de confiance, comme dans la pièce de Calderon? Souffre-t-il de ne pas croire en Dieu, nom possible de l'impossible? Je ne crois pas : il a répondu à la béance entrevue en lui donnant le nom des extraterrestres, figures diabolisées, en lieu et place du nom du Dieu. C'est d'ailleurs la raison qui explique la similitude foncière entre Mulder et sa partenaire. Scully n'ignore pas le réel, mais son voile. Le voile dont elle a choisi de draper ce réel, qui le nomme, le justifie et le fait verser du côté du sens, c'est la religion catholique à laquelle elle tient. Et qui la tient. Ainsi les deux personnages ont-ils une complicité foncière, même si leurs modes de croyance diffèrent et même si l'adhésion de Mulder à son propre système de croyances est plus ténue, ce qui explique d'ailleurs l'errance du personnage, sa mélancolie profonde.

\section{Le récit du réel}

Ainsi, il convient de distinguer nettement dans cette série l'histoire et le récit qui nous en est proposé. Il y a en effet une différence nette entre ce que croit Mulder et ce que dit la série. Pour Mulder, adepte de la théorie du complot, « rien n'est tel qu'il paraît être. Les apparences sont donc toujours trompeuses. Tout est masque, donc à démasquer. » (Taguieff, 2008, p. 59) ${ }^{3}$ Sous le masque est la vérité ; elle est dite, et nommée. La série, en revanche, par ses modalités narratives et le jeu syncrétique, suspend la réponse, en insérant des épisodes qui ne se rangent pas sous la bannière thématique du complot et en privilégiant l'effet d'inachèvement. Mulder croit en la

3 Selon le troisième des quatre principes de structuration du complot, formalisés par Pierre-André Taguieff (p. 59). 
possibilité d'un sens dernier, croyance que la série dément de par sa structure même.

Philippe Forest, dans l'avant-propos de son ouvrage Le roman, le réel et autres essais, rappelle la double censure qui barre le concept de réel :

D’un côté, le néo-naturalisme (tel qu'il domine dans les formes hégémoniques du roman commercial et de la « world fiction ») manque le réel en prétendant pouvoir offrir du monde une représentation positive de laquelle se trouve proscrite toute réflexion du texte sur lui-même et sur l'impossible dont il procède. De l'autre, le néo-formalisme (que revendique toute une part de la création romanesque et poétique nouvelle), solidaire d'une esthétique de la simulation et du virtuel, congédie ce même réel au nom d'une conception de la littérature détachée du monde, s'enchantant d'elle-même et s'assumant comme un exercice ludique et ironique suffisant. (p.13)

La série X-Files revisite les modalités traditionnelles de narration dans le domaine du fantastique, c'est un fait. Pourtant par cette disjonction entre les discours (du personnage et de la narration), la série dépasse les deux écueils présentés par Philippe Forest. La mise en récit, loin d'opter pour un épuisement qui la cantonnerait à l'un ou l'autre de ces discours, fait le pari de les unir. Tout d'abord, la série peut être dite néonaturaliste, non pas dans le sens où elle offrirait « du monde une représentation positive », mais où elle propose une critique de ce monde en plaçant en tension réel et réalité. Le surcroît de cette dernière, son excès même venant masquer le réel, par l'afflux des systèmes symboliques, est incessamment troué, on l'a vu, par ce même réel qu'elle tente de représenter avec les moyens offerts par l'imaginaire. La série assume dans ce contexte une position vigoureusement polémique en même 
temps que, par les enquêtes opiniâtres au service des « affaires non classées » qu'elle met en scène, elle entreprend de creuser le négatif répudié par le puritanisme contemporain. Sa lutte est donc à comprendre comme une opposition franche envers toute tentative d'appauvrissement du réel au profit du surcroît de réalité (qu'exemplifie l'agent Colton, qui œuvre à son établissement personnel dans le troisième épisode de la première saison). La série, qui lutte pour accorder au réel la place qu'il mérite, réintroduit ainsi un principe de négativité et parie sur l'ombre et sa possible et nécessaire fécondité.

Cette dimension se double, dans la série, d'un néoformalisme qui, paradoxalement, concourt au même but. Je n'en donnerai qu'un exemple. À la fin de L'enlèvement, Scully, qui n'est pas dupe quant aux motifs qui suscite l'investissement de Mulder dans cette enquête, écoute les enregistrements d'une hypnose de Mulder. Celui-ci raconte que sa sœur l'appelle au secours. Une voix lui dit de ne pas avoir peur, que sa sœur reviendra. «Vous croyez ce que dit cette voix? » — « Je veux y croire », répond Mulder. Dans le même temps, à l'image, on le voit en pleurs dans une église. Certes, ces propos apparaissent comme un commentaire de la tragédie vécue par le personnage, mais ils mettent aussi en question le principe même du récit. En effet, Mulder, au cours de ses enquêtes, affronte tout aussi bien le réel que le récit qui en résulte. De ce réel, on ne peut obtenir que la fiction. Et c'est là tout son drame, mais ce drame, à travers l'immersion fictionnelle, devient partageable. Ainsi, la série, qui place en tension réel, imaginaire et symbolique, pense dans le même temps ses propres conditions de possibilité : toute histoire est à la fois traitement du réel (au sens d'une tentative de nomination), mise en œuvre de l'imaginaire et médiatisation du symbolique. Au fond, qu'est Mulder, sinon un 
lecteur ou un spectateur du monde, dimension que métaphorise sa fonction, qui essaie, trop rapidement sans doute, d'en démêler les instances et de leur assigner enfin une place juste?

\section{Bibliographie}

FoREST, Philippe. (2007), Le Roman, le réel et autres essais, Allaphbed 3, Nantes, Éditions Cécile Defaut.

ISER, Wolfgang. (1997), L'Acte de lecture, théorie de l'effet esthétique, Bruxelles, Mardaga, coll. "Philosophie et langage $»$.

LACAN, Jacques. (2005), Le Séminaire Livre XXIII, Le sinthome, Paris, Seuil.

POMMIER, Gérard. (2004), Qu'est-ce que le réel?, Paris, Érès, coll. « Point hors ligne».

TAGUIEFF, Pierre-André. (2008), L'Imaginaire du complot mondial, aspects d'un mythe moderne, Paris, Mille et une nuits.

ZIZEK, Slavoj. (2007), Bienvenue dans le désert du réel, Paris, Flammarion, coll. « Champs ». 


\title{
Résumé
}

Dans la série $X$-Files, la réalité est pensée, de manière lacanienne, comme une surabondance illusoire (paradoxalement d'une grande pauvreté) et comme un leurre, que crée et vectorise le langage et qu'intensifie le désir de croire aux témoignages de nos sens. Or, parce que le personnage de Mulder cherche à nommer ce réel, il le manque et est voué dès lors à une "passion des semblants", envers de la "passion du réel » définie par Zizek. C'est dès lors la série - par son inachèvement et ses ellipses - qui prendra en charge cette rétivité foncière du réel à la nomination.

\begin{abstract}
Reality is, in the series $X$-Files, thought in a Lacanian manner, as an illusory abundance (paradoxically, of a great poverty) and as a decoy created and vectorized by language and intensified by the desire to believe the testimony of our senses. However, because the character of Mulder tries to name the "real", he misses it and is therefore doomed to a "passion of semblance", opposite of the "real passion" defined by Zizek. It is therefore the series - through its incompleteness and ellipses - that will confront this resistance of the "real" to denomination.
\end{abstract}

